\title{
Pretransplant locoregional therapy for hepatocellular carcinoma: encouraging but insufficient
}

\author{
Seong Hoon Kim, Eung Chang Lee, Sang Jae Park \\ Organ Transplantation Center/Center for Liver Cancer, National Cancer Center, Goyang-si, Gyeonggi-do, Republic of Korea \\ Correspondence to: Seong Hoon Kim, MD, PhD. Organ Transplantation Center/Center for Liver Cancer, National Cancer Center, 323 Ilsan-ro, \\ Ilsandong-gu, Goyang-si, Gyeonggi-do 10408, Republic of Korea. Email: kshlj@hanmail.net; kshlj@ncc.re.kr. \\ Provenance: This is an invited Editorial commissioned by Editor-in-Chief Yilei Mao (Department of Liver Surgery, Peking Union Medical College \\ Hospital, Chinese Academy of Medical Sciences, Beijing, China). \\ Comment on: Agopian VG, Harlander-Locke MP, Ruiz RM, et al. Impact of Pretransplant Bridging Locoregional Therapy for Patients With \\ Hepatocellular Carcinoma Within Milan Criteria Undergoing Liver Transplantation: Analysis of 3601 Patients From the US Multicenter HCC \\ Transplant Consortium. Ann Surg 2017;266:525-35.
}

Submitted Jan 07, 2018. Accepted for publication Jan 24, 2018.

doi: $10.21037 /$ hbsn.2018.01.08

View this article at: http://dx.doi.org/10.21037/hbsn.2018.01.08

Despite in the era of liver transplantation (LT) widely performed as an established curative treatment for hepatocellular carcinoma (HCC), the scarcity of liver donors has still been a well-nigh insurmountable barrier to access of LT. So, the bridging locoregional therapy (LRT) that treats HCC during the waiting time prior to LT emerged as an attractive strategy to reduce the risk of tumor progression and waitlist dropout, which came to an international consensus statement regarding the waitlist management of HCC patients (1). However, the actual advantage for LRT has not been clearly demonstrated in terms of recurrence and survival.

Considering the risk of waitlist dropout, a randomized controlled trial comparing LRT with no LRT in patients awaiting LT may not be justified. Furthermore, existing data have mostly been collected from retrospective, single-center experiences of limited sample sizes, making it difficult to draw solid conclusions.

The article by Agopian et al. (2), recently published in Annals of Surgery from a multi-center study in US, brings up again the effect of pretransplant bridging LRT on HCC recurrence and survival after LT in patients meeting Milan criteria. The bottom line of the findings was that bridging LRT in HCC patients within Milan criteria does neither improve post-LT survival nor reduce HCC recurrence in patients who fail to achieve complete pathologic response (cPR). And the interesting point about these findings was that the two factors, the need for increasing LRTs and poor response of alpha-fetoprotein to LRT, were independent predictors for post-LT recurrence.

As of now, this study is the largest ever reported about the outcomes of LRT before LT in patients for HCC within Milan criteria, recruiting an overwhelming number of patients whose data was incorporated from 20 centers involving 10 out of 11 United Network for Organ Sharing (UNOS) regions, which could possibly represent clinical outcomes and practice across the US. And the detailed pathologic data on explant livers reinforce the solidity and scope of a study design.

The key finding to this study was that recurrence was higher in recipients receiving LRT and not achieving $\mathrm{cPR}$ than in those who received no LRT or those receiving LRT and showing $\mathrm{cPR}$, suggesting the pathologic response to pre-LT LRT as an important surrogate for more aggressive tumor biology. Assuringly the result was derived by a multivariate analysis controlling for pretransplant (diagnosis, model for end-stage liver disease score, alpha-fetoprotein) and pathologic (tumor diameter, differentiation, vascular invasion) characteristics. In other words, in patients not achieving cPR, LRT may be potential culprit for more aggressive tumor behavior behind recurrence, which sends an important and intriguing message that the tumor had better be left untouched till LT. This study provides beneficial and interesting information that can assist in deciding on whether to perform LRT in LT patients for HCC or not.

However, it is difficult to know which patients receiving 
LRT will achieve cPR in the actual clinical setting, because cPR cannot be confirmed without explant pathology before LT. Nonnegligible are the chances that pre-LT needle biopsy can increase tumor seeding and cause bleeding especially in cirrhotic patients, which reasonably can make the use of pre-LT pathologic characteristics for patient selection debatable. Biomarkers as predictors of HCC recurrence have been reported to include tumor markers (alpha-fetoprotein, des-gamma-carboxy prothrombin), fluorine-18-fluorodeoxyglucose $\left({ }^{18} \mathrm{~F}-\mathrm{FDG}\right)$ positron emission tomography (PET) and systemic inflammatory markers (3). In addition to these factors, more refined evaluation criteria for radiologic response to each LRT could be incorporated as key parameters in building a predictive model that can assess the response to LRT nearly as accurately as explant pathology. To this purpose, future studies should focus on refinement of selection criteria for LRT in HCC patients waiting LT in terms of tumor biology and intrahepatic tumor burden (number and size), although the authors of this study didn't make any comments in terms of radiologic imaging response to pre-LT LRT.

A note of caution needs to be added in interpreting the study result that more LRT treatments independently predicted post-LT recurrence. Generally the increasing number of LRT was highly likely to be due either to long waiting time or to tumor progression. The specific data on the outcomes according to the number of LRT in the patients who achieved $\mathrm{cPR}$ are necessary to support the claims of the paper. So further detailed analyses were warranted to endorse the conclusion that LRT should not be done in some groups of HCC patients.

Currently, the Milan criteria are a widely accepted set of criteria used to justify LT for HCC by demonstrating the long-term results to be comparable to those of LT for non-malignant conditions (4). The question may then arise, "Whatever benefit can pre-LT LRT have practically in the patient selection within the Milan criteria if not for preventing tumor progression for a long waiting time period, but for selecting favorable tumor biology?”. The authors concluded that LRT can be served as a surrogate underlying tumor biology for prioritization of HCC LT candidates. Surely, some follow-up periods are required to differentiate patients with favorable tumor biology. If the tumor should progress during the period, the backfiring of the very LRT would end up having serious disadvantages: LRT-induced accelerated tumour cell repopulation, delay in performing a potentially curative LT thus risking localized or metastatic spread if the LRT is ineffective, leading to waitlist drop out. So, the actual outcomes of LRT, beneficial or harmful, need to be evaluated on an intent-to-treat analysis of all listed patients.

In short, based on the results of the present multicenter large study, pre-LT LRT can be an option as one possible way to increase the pCR rate, achieving good oncologic outcomes after LT, although the data seems insufficient to assess the effects of LRT in the entire cohort of HCC patients listed, and the optimal treatment choice and frequency out of a number of different LRT options have not been determined. Hopefully, additional LRT studies of high quality could be undertaken in HCC patients beyond the Milan criteria or listed for LDLT.

\section{Acknowledgements}

None.

\section{Footnote}

Conflicts of Interest: The authors have no conflicts of interest to declare.

\section{References}

1. Clavien PA, Lesurtel M, Bossuyt PM, et al. Recommendations for liver transplantation for hepatocellular carcinoma: an international consensus conference report. Lancet Oncol 2012;13:e11-22.

2. Agopian VG, Harlander-Locke MP, Ruiz RM, et al. Impact of Pretransplant Bridging Locoregional Therapy for Patients With Hepatocellular Carcinoma Within Milan Criteria Undergoing Liver Transplantation: Analysis of 3601 Patients From the US Multicenter HCC Transplant Consortium. Ann Surg 2017;266:525-35.

3. Lai Q, Lerut JP. Hepatocellular cancer: how to expand safely inclusion criteria for liver transplantation. Curr Opin Organ Transplant 2014;19:229-34.

4. Mazzaferro V, Regalia E, Doci R, et al. Liver transplantation for the treatment of small hepatocellular carcinomas in patients with cirrhosis. $\mathrm{N}$ Engl J Med 1996;334:693-9.

Cite this article as: Kim SH, Lee EC, Park SJ. Pretransplant locoregional therapy for hepatocellular carcinoma: encouraging but insufficient. HepatoBiliary Surg Nutr 2018;7(2):136-137. doi: 10.21037/hbsn.2018.01.08 